\title{
Study of microbial spread when using multiple-use nasal anaesthetic spray*
}

\author{
Mamun Rashid and Yakubu G. Karagama \\ Department of Otolaryngology, Tameside Hospital, Ashton-under-Lyne, United Kingdom
}

SUMMARY Background: Current economic constraints have led to the emergence of reusable anaesthetic sprays with replacement nozzles (Xylocaine) as an alternative to disposable anaesthetic solutions (Co-phenylcaine) for rhinological procedures. The former costs $£ 191$ per year versus $£ 10,761$ for the latter, at equivocal doses. However, research regarding the sterility of such instruments is limited. The aim of this in vitro study was to determine whether bottles of Xylocaine could pose a risk of cross-infection to patients.

Methods: Two techniques were used. The first was to introduce nozzles into methylene blue and fluorescein dyes, and then analysing the anaesthetic solutions using spectrophotometry for assessment of colour change indicating retrograde contamination. The second method involved spraying Xylocaine into cultures of Staphylococcus aureus with concurrent nozzle changes. This was repeated over a 36-day-period. Sterility checks were performed on the Xylocaine before and after inoculation into the culture.

Results: None of the anaesthetic solutions showed the presence of dyed saline following spectrophotometric analysis. No S. aureus was isolated from any of the 30 spray bottles cultures over the 36-day trial period.

Conclusion: It was demonstrated that using the Xylocaine spray with disposable nozzles for each patient should not pose a cross infection risk to patients.

Key words: nasal spray, anaesthetic, cross infection, sterility

\section{BACKGROUND}

Lidocaine and Phenylephrine Spray (LPS) is supplied as a single patient nasal preparation, which is effective on mucous membranes and dominates current practice as the choice for topical nasal anaesthesia for common rhinological procedures. Each pump dispenser is reported to deliver $6.5 \mathrm{mg}$ lidocaine and $0.65 \mathrm{mg}$ phenylephrine per spray ${ }^{(1)}$. More recently, Xylocaine Spray (XS) has been developed by manufacturers as a multi-use bottle, which can be used with disposable nozzles for multiple patients. It is a prepackaged, commercial solution. Each dose of XS contains Lidocaine and Phenylephrine, Eur $10 \mathrm{mg}$, ethanol, Macrogol 400, essence of banana, menthol natural, saccharin and purified water ${ }^{(2)}$. A disposable plastic nozzle is attached to the anaesthetic bottle, the spray is administered nasally to patients and the nozzle is discarded. These steps are repeated for each patient. LPS comes as a $2.5 \mathrm{ml}$ preparation at a cost of $£ 10.02$ per bottle whilst XS at $50 \mathrm{mls}$ costs $£ 3.68$ per bottle; in other words the latter is potentially capable of yielding 20 times the dose of the former ${ }^{(3)}$. In our department in a busy district general hospital, 1074 bottles of LPS were ordered by the pharmacy department at a cost of $£ 10,761$ for the year. The equivalent cost of XS was £ 191. The fiscal incentive for adopting a multi-use regime is thus eminently apparent. However, the potential risk of patient crosscontamination with multiple uses is less clear and there are no prior studies to our knowledge that has addressed this significant issue. The sterility of ophthalmic drugs dispensed from spray bottles has previously been assessed with the authors of one study concluding that there appears to be minimal risk of instilling contaminated diagnostic drugs using the spray method when a single drug is stored in a spray bottle ${ }^{(4)}$. Our paper sets out not only to ascertain the sterility of anaesthetic solutions over a time period, but also to assess the potential contamination risk of anaesthetic fluid from the backflow of sprayed solution from the patient into the anaesthetic solution container. 
The aim of the study was to determine whether using a nasal Xylocaine spray with replacement nozzles presents a cross infection risk to patients.

\section{MATERIAL AND METHODS}

Two techniques were used to assess microbial spread.

\section{Method 1. Spectrophotometer analysis}

Spectrophotometric analysis of the Xylocaine took place prior to application of nozzles dipped in the dyed solutions to establish baseline colour (Figure 1). Subsequently nozzles were inserted into containers with methylene blue and fluorescein dyes separately with the Xylocaine solution re-analysed for any colour change (Figure 2). The containers were kept away from light prior to the experiment to ensure uniformity of baseline colours.

\section{Method 2. Culture analysis}

A standard inoculating culture was prepared using one colony of $S$. aureus into a $10 \mathrm{ml}$ nutrient broth, which was then incubated aerobically at $37^{\circ} \mathrm{C}$ for 24 hours. A $100 \mathrm{ml}$ bottle of sterile water was used combined with $100 \mu$ of overnight culture to prepare a 1:1000 dilution to give an organism count of $10^{8} /$ $\mathrm{L}$ or greater.

The nozzles were placed into the Xylocaine bottle and an application of one spray onto a blood plate was used to assess pre-sterility. The attached nozzle was then submerged directly into the $100 \mathrm{ml}$ culture bottle with the open neck sealed with parafilm and then 1 spray of Xylocaine was applied into the culture. After discarding the first nozzle and applying a second nozzle, a further single spray was applied to a blood plate to assess post-sterility. The blood agar plates were then incubated for 48 hours and bacterial growth was checked using a CLED dip strip plate. This was repeated at daily intervals over a 36-day period. The same steps were also repeated using 30 spray applications to determine if multiple sprays yielded any difference in bacterial growth. After completing the 36-day

Table 1. Spectrophotometer analysis - Methylene Blue.

\begin{tabular}{ccc}
\hline Batch No. & Pre - Dye Reading & Post - Dye Reading \\
\hline 1 & 0.002 & 0.005 \\
2 & 0.004 & 0.002 \\
3 & 0.007 & 0.004 \\
4 & 0.004 & 0.006 \\
5 & 0.005 & 0.008 \\
\hline
\end{tabular}

* Note each batch is comprised of 10 samples with the reading recorded as the mean result of each set. testing protocol described above, the procedure was also repeated several times over the course of 1 day for both the 1 and 30 spray bottles. The procedure was repeated 6 times over a 5.5 hour period.

\section{RESULTS}

Results (1) spectrophotometer analysis

The results are presented in Table 1 and 2.

Results (2) culture analysis

\section{1 spray inoculation}

Daily inoculation: In the pre-sterility cultures, over the 36-day period, in all the sterility cultures, no growth was seen. On the other hand, in the post sterility cultures, on day 9 , the sterility culture contained 8 colonies of $S$. aureus. All of the remaining cultures did no grow (Table 3 ).

\section{Multiple inoculation over a one day period}

The procedure was repeated 6 times over 5.5 hours. Each procedure consisted of 30 sprays. In both the pre sterility cultures and the post sterility cultures, no growth was visible.

Diluting an overnight culture of $S$. aureus gave a bacterial count of $10^{8}$.

\section{Nozzle sterility checks}

No pathogenic micro-organisms including $S$. aureus were isolated from any of the nozzle sterility checks, but some low numbers of bacteria normally associated with skin carriage were isolated.

\section{0 spray inoculation}

Daily inoculation: in the pre-sterility cultures, over the 36-day period, in all of the sterility checks, no growth was seen. This was also the case with the post-sterility cultures (Table 4).

\section{DISCUSSION}

Changes in the way the anaesthetic spray Xylocaine is administered has raised concerns regarding cross infection of nasal
Table 2. Spectrophotometer analysis - Fluorescein.

\begin{tabular}{ccc}
\hline Batch No.* & Pre - Dye Reading & Post - Dye Reading \\
\hline 1 & 0.006 & 0.005 \\
2 & 0.005 & 0.002 \\
3 & 0.007 & 0.008 \\
4 & 0.004 & 0.004 \\
5 & 0.003 & 0.005 \\
\hline
\end{tabular}

* Note each batch is comprised of 10 samples with the reading recorded as the mean result of each set. 
Table 3. Daily Inoculation 1 Spray into S. aureus Culture Bottle.

\begin{tabular}{|c|c|c|c|c|c|}
\hline Date & $\begin{array}{c}\text { Spray Sterility Check } \\
\text { (pre inoculation) } 48 \\
\text { hrs }\end{array}$ & $\begin{array}{l}\text { Nozzle sterility Check } \\
\qquad 1^{\text {st }}\end{array}$ & $\begin{array}{l}\text { Nozzle Sterility Check } \\
\qquad 2^{\text {nd }}\end{array}$ & Bacterial count/l & $\begin{array}{l}\text { Post inoculation } \\
\text { Spray culture } 48 \text { hrs } \\
\text { (fresh nozzle) }\end{array}$ \\
\hline Day 1 & NG & SC STE/DIPS & NG & $10^{8}$ & NG \\
\hline Day 3 & NG & SC skin & NG & $10^{8}$ & NG \\
\hline Day 9 & NG & SC skin & NG & $10^{8}$ & $\begin{array}{l}8 \text { colonies } \\
\text { S. aureus }\end{array}$ \\
\hline Day 17 & NG & NG & SC skin & $10^{8}$ & NG \\
\hline Day 19 & NG & NG & SC skin & $10^{8}$ & NG \\
\hline Day 24 & NG & NG & NG & $10^{8}$ & NG \\
\hline Day 34 & NG & NG & NG & $10^{8}$ & NG \\
\hline \multicolumn{6}{|c|}{1 spray bottle multiple inoculation over a 1 day period ( 30 sprays) } \\
\hline 11.15 & NG & 1 col skin & NG & $10^{8}$ & NG \\
\hline 11.45 & NG & NG & NG & $10^{8}$ & NG \\
\hline 12.30 & NG & $1 \mathrm{col}$ skin & NG & $10^{8}$ & NG \\
\hline 13.00 & NG & $1 \mathrm{col}$ skin & NG & NG & NG \\
\hline 14.30 & NG & 1 col skin & 2 cols skin & NG & NG \\
\hline 17.00 & NG & 2 cols & NG & NG & NG \\
\hline
\end{tabular}

Bacterial count: $>25$ colonies $=10^{8} / \mathrm{L}, \mathrm{NG}=$ No Growth, col $=$ colony, $\mathrm{SC}=$ scanty

Table 4. Daily Inoculation 34 Spray into S. aureus Culture Bottle.

\begin{tabular}{|c|c|c|c|c|c|}
\hline Date & $\begin{array}{l}\text { Spray sterility check } \\
\text { (pre inoc) } 48 \text { hrs inc }\end{array}$ & $\begin{array}{l}\text { Nozzle sterility check } \\
\qquad 1^{\text {st }}\end{array}$ & $\begin{array}{l}\text { Nozzle sterility check } \\
\qquad 2^{\text {nd }}\end{array}$ & Bacterial count & $\begin{array}{l}\text { Post inoculation } \\
\text { spray culture } 48 \text { hrs } \\
\text { (fresh nozzle) }\end{array}$ \\
\hline Day 1 & $\mathrm{NG}$ & 2 cols skin & 3 cols skin & $10^{8}$ & $\mathrm{NG}$ \\
\hline Day 3 & $\mathrm{NG}$ & $1 \mathrm{col}$ skin & 3 cols-asp fu & $10^{8}$ & $\mathrm{NG}$ \\
\hline Day 9 & $\mathrm{NG}$ & 1col skin & $\mathrm{NG}$ & $10^{8}$ & $\mathrm{NG}$ \\
\hline Day 17 & $\mathrm{NG}$ & $1 \mathrm{col}$ skin & $\mathrm{NG}$ & $10^{8}$ & $\mathrm{NG}$ \\
\hline Day 19 & $\mathrm{NG}$ & $\mathrm{NG}$ & 3 cols skin & $10^{8}$ & $\mathrm{NG}$ \\
\hline Day 24 & NG & SC skin & SC skin & $10^{8}$ & NG \\
\hline Day 34 & NG & $\mathrm{NG}$ & NG & $10^{8}$ & NG \\
\hline \multicolumn{6}{|c|}{30 spray bottle multiple inoculation over a one day period ( 30 sprays) } \\
\hline 11.15 & NG & SC skin & SC skin & $10^{8}$ & $\mathrm{NG}$ \\
\hline 11.45 & NG & SC skin & SC skin & $10^{8}$ & NG \\
\hline 12.30 & $\mathrm{NG}$ & SC skin & SC skin & $10^{8}$ & $\mathrm{NG}$ \\
\hline 13.00 & $\mathrm{NG}$ & SC skin & SC skin & $10^{8}$ & $\mathrm{NG}$ \\
\hline 14.30 & $\mathrm{NG}$ & SC skin & SC skin & $10^{8}$ & $\mathrm{NG}$ \\
\hline 17.00 & $\mathrm{NG}$ & SC skin & SC skin & $10^{8}$ & $\mathrm{NG}$ \\
\hline
\end{tabular}


flora between patients. The condition in which the Xylocaine is administered was recreated in the laboratory using a $10^{8}$ culture of $S$. aureus, as this is the organism most commonly carried in the nasal passages of patients ${ }^{(5)}$. Inoculating the Xylocaine directly into the $S$. aureus culture daily over a 36-day period using 1 or 30 sprays gave negative cultures with the exception of day 9 - one spray post inoculation sterility check. Subsequent pre- and post inoculation sterility did not show any growth. This isolated result may be a result of laboratory contamination via aerosol production. Repeating the procedure 6 times over a 5.5 hour period also yielded no culture growth. Patients are sprayed once with the Xylocaine, repeating the procedure with 30 sprays demonstrated that the procedure was not a risk to patients in doses far in excess of those administered.

The Xylocaine and the disposable tips are stored at room temperature prior to use. All of the pre inoculation sterility checks did not show any growth, the medium therefore can be stored at room temperature without presenting a cross infection risk to patients.

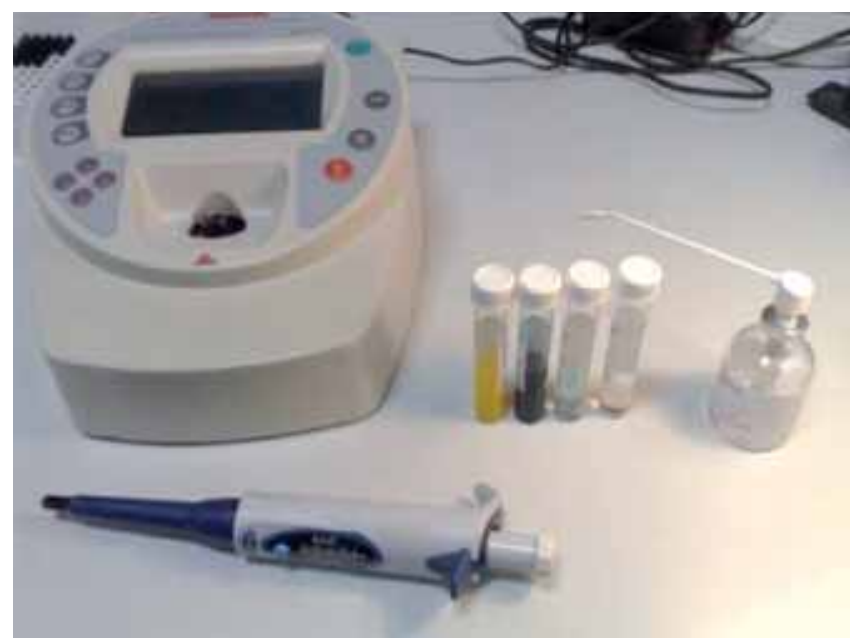

Figure 1. Image of the spectrophotometer together with Xylocaine spray and dyes.

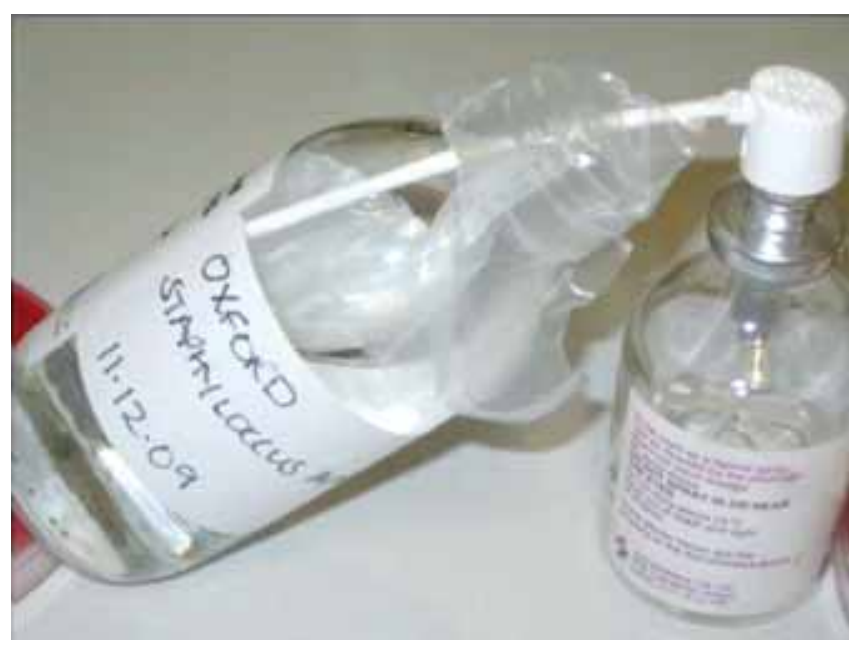

Figure 3. Image of nozzle into staph solution set up.
The nozzles are not sterile. Nozzle sterility checks did not show any growth or only scanty skin flora and therefore do not appear to pose a cross infection risk. The dye experiment also did not demonstrate any evidence of contamination.

\section{CONCLUSION}

Using multiple use anaesthetic spray with disposable nozzles for each patient should not pose a cross infection risk through backflow to the bottles.

\section{ACKNOWLEDGEMENTS}

Cathy Hatch, Senior Microbiology Technician, Tameside Hospital and Tony Sivner, Chief Pharmacist, Tameside Hospital.

\section{AUTHORSHIP CONTRIBUTIONS}

Both authors have played a significant contribution to the paper. Mr Karagama designed the study including methodology and analysis, Mr Rashid was involved in developing the methodology, was involved in data collection, and both were involved in the drafting and analysis aspects of the paper.

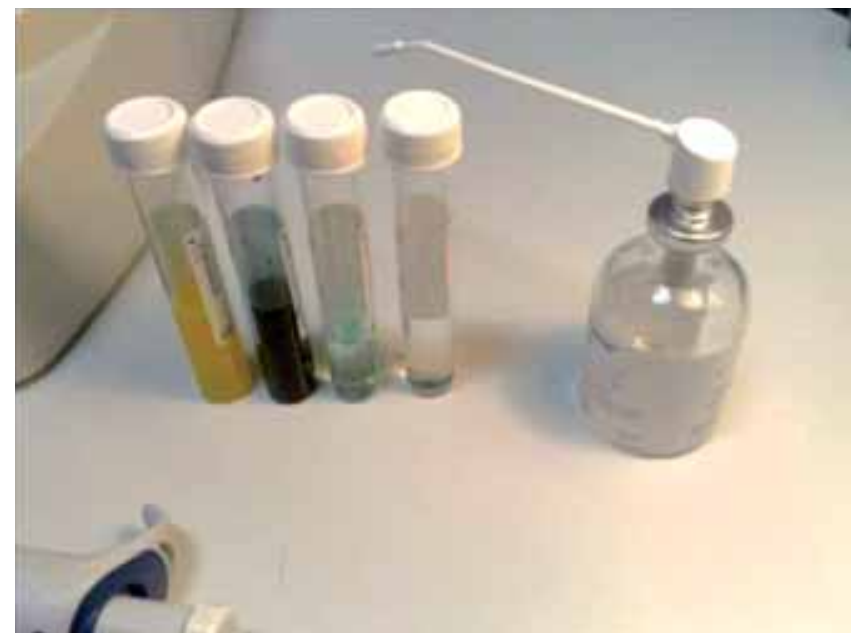

Figure 2. Image of the dyes close up.

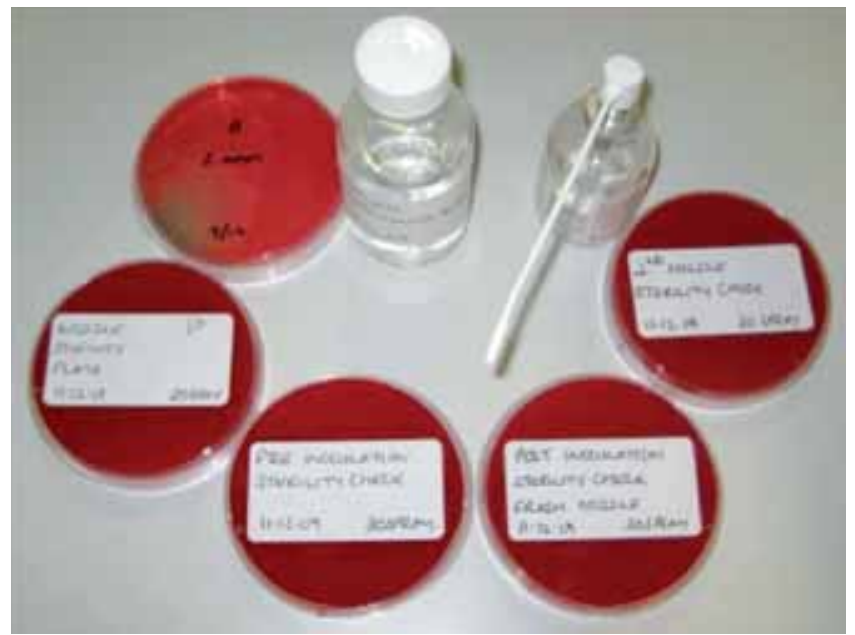

Figure 4. Image of blood agar plates at various check points. 


\section{CONFLICTS OF INTEREST}

None declared.

Mamun Rashid

\section{REFERENCES}

1. EPG Online. Lidocaine and Phenylephrine topical solution [Internet]. 2010 [Cited 2010 May 19]. Available from http://www. epgonline.org/viewdrug.cfm/drugId/DR000251/language/LG0001/ drugname/Lidocaine-and-phenylephrine-topical solution

2. Express Chemist. Xylocaine Spray 50mls [Internet]. 2010 [Cited 2010 May 19]. Available from http://www.expresschemist.co.uk/ Xylocaine-Spray- $50 \mathrm{ml}$

3. BNF [Internet]. 2010 [Cited Jun 12]. Available from http://bnf.org/ bnf/index

4. Kim, G, Fern, K, and Perrigin, J. Sterility of ophthalmic drugs dispensed from spray bottles. Optom Vis Sci. 1997; 74: 865-867.

5. Ythier M, Entenza J, Bille J, Vandenesch F, Moreillon P, Sakwinska, O. Natural variability of in vitro adherence to fibrinogen and fibronectin does not correlate with in vivo infectivity of Staphylococcus aureus. Infect Immun 2010; 78: 1711-1716.

\section{ADVERTISEMENT}

International Federation Facial Plastic Surgery Societies (IFFPSS)

VII International Congress

Congress President: Pietro Palma, MD

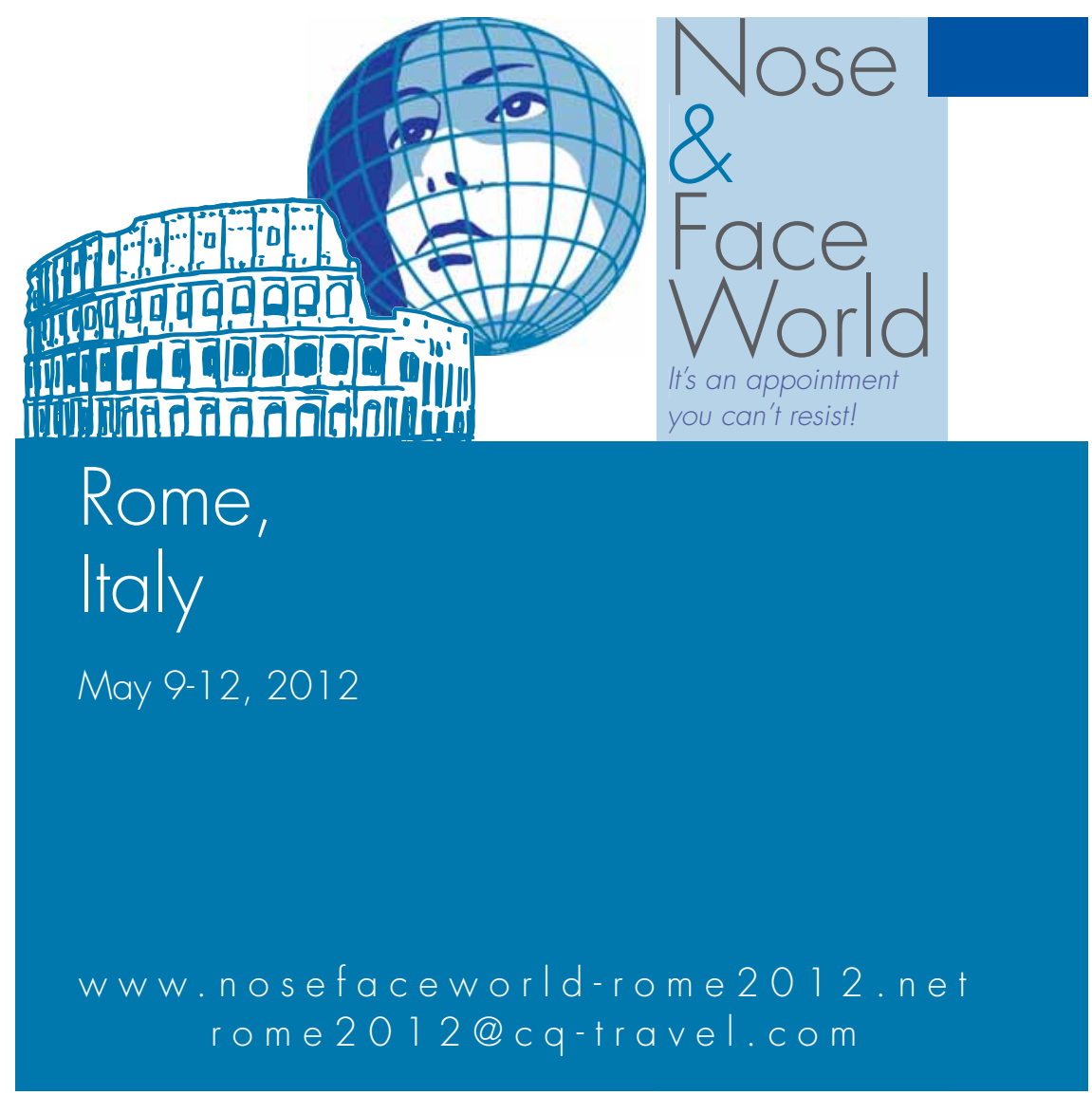

